This is the accepted version of the following article:

Soler M., Mesa-Antunez P., Estevez M.-C., Ruiz-Sanchez A.J., Otte M.A., Sepulveda B., Collado D., Mayorga C., Torres M.J ., Perez-Inestrosa E., Lechuga L.M.. Highly sensitive dendrimer-based nanoplasmonic biosensor for drug allergy diagnosis. Biosensors and Bioelectronics, (2015). 66. : 115 - . 10.1016/j.bios.2014.10.081,

which has been published in final form at https://dx.doi.org/10.1016/j.bios.2014.10.081 (c) https://dx.doi.org/10.1016/j.bios.2014.10.081. This manuscript version is made available under the CC-BY-NC-ND 4.0 license http://creativecommons.org/licenses/by-nc-nd/4.0/ 


\section{Author's Accepted Manuscript}

Highly sensitive dendrimer-based nanoplasmonic biosensor for drug allergy diagnosis

Maria Soler, Pablo Mesa-Antunez, M.-Carmen Estevez, Antonio Jesus Ruiz-Sanchez, Marinus A. Otte, Borja Sepulveda, Daniel Collado, Cristobalina Mayorga, Maria Jose Torres, Ezequiel Perez-Inestrosa, Laura M. Lechuga

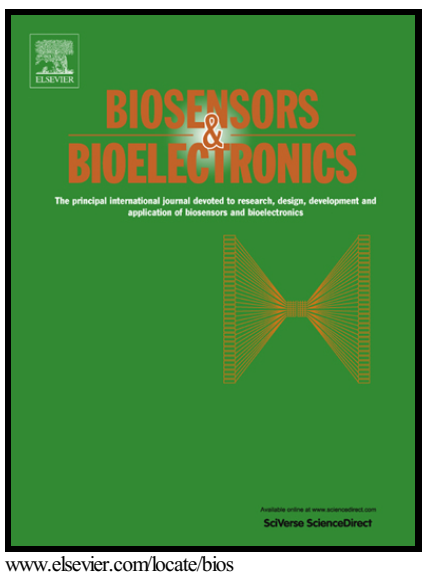

PII: $\quad$ S0956-5663(14)00883-5

DOI: $\quad$ http://dx.doi.org/10.1016/j.bios.2014.10.081

Reference: BIOS7268

To appear in: Biosensors and Bioelectronic

Received date: 2 September 2014

Revised date: 20 October 2014

Accepted date: 21 October 2014

Cite this article as: Maria Soler, Pablo Mesa-Antunez, M.-Carmen Estevez, Antonio Jesus Ruiz-Sanchez, Marinus A. Otte, Borja Sepulveda, Daniel Collado, Cristobalina Mayorga, Maria Jose Torres, Ezequiel Perez-Inestrosa and Laura M. Lechuga, Highly sensitive dendrimer-based nanoplasmonic biosensor for drug allergy diagnosis, Biosensors and Bioelectronic, http://dx.doi.org/10.1016/j.bios.2014.10.081

This is a PDF file of an unedited manuscript that has been accepted for publication. As a service to our customers we are providing this early version of the manuscript. The manuscript will undergo copyediting, typesetting, and review of the resulting galley proof before it is published in its final citable form. Please note that during the production process errors may be discovered which could affect the content, and all legal disclaimers that apply to the journal pertain. 


\title{
Highly Sensitive Dendrimer-based Nanoplasmonic Biosensor for Drug Allergy Diagnosis
}

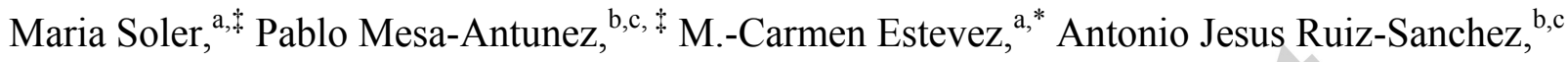 \\ Marinus A. Otte, ${ }^{a}$ Borja Sepulveda, ${ }^{a}$ Daniel Collado,,${ }^{\text {bc }}$ Cristobalina Mayorga, ${ }^{\text {,d }}$ Maria Jose \\ Torres, ${ }^{c, e}$ Ezequiel Perez-Inestrosa, ${ }^{\text {b,c }}$ Laura M. Lechuga ${ }^{\text {a }}$ \\ ${ }^{a}$ Nanobiosensors and Bioanalytical Applications Group, Institut Català de Nanociència $i$ \\ Nanotecnologia (ICN2), CSIC and CIBER-BBN, 08193 Bellaterra (Barcelona), Spain. \\ ${ }^{b}$ University of Malaga, IBIMA, Department of Organic Chemistry 29071-Malaga, Spain. \\ ${ }^{c}$ Andalusian Centre for Nanomedicine and Biotechnology-BIONAND, Parque Tecnologico de \\ Andalucia, 29590 Malaga, Spain. \\ ${ }^{d}$ Research Laboratory, IBIMA, Regional University Hospital of Malaga, UMA, 29009 Malaga, \\ Spain \\ ${ }^{e}$ Allergy Unit, IBIMA, Regional University Hospital of Malaga, UMA, 29009 Malaga, Spain \\ $\$$ These authors contributed equally \\ *Corresponding author: mcarmen.estevez@cin2.es
}


ABSTRACT A label-free biosensing strategy for amoxicillin (AX) allergy diagnosis based on the combination of novel dendrimer-based conjugates and a recently developed nanoplasmonic sensor technology is reported. Gold nanodisks were functionalized with a custom-designed thiolending- polyamido-based dendron (d-BAPAD) peripherally decorated with amoxicilloyl (AXO) groups (d-BAPAD-AXO) in order to detect specific IgE generated in patient's serum against this antibiotic during an allergy outbreak. This innovative strategy, which follows a simple one-step immobilization procedure, shows exceptional results in terms of sensitivity and robustness, leading to a highly-reproducible and long-term stable surface which allows achieving extremely low limits of detection. Moreover, the viability of this biosensor approach to analyze human biological samples has been demonstrated by directly analyzing and quantifying specific anti-AX antibodies in patient's serum without any sample pretreatment. An excellent limit of detection (LoD) of $0.6 \mathrm{ng} / \mathrm{mL}$ (i.e. $0.25 \mathrm{kU} / \mathrm{L}$ ) has been achieved in the evaluation of clinical samples evidencing the potential of our nanoplasmonic biosensor as an advanced diagnostic tool to quickly identify allergic patients. The results have been compared and validated with a conventional clinical immunofluorescence assay (ImmunoCAP test), confirming an excellent correlation between both techniques. The combination of a novel compact nanoplasmonic platform and a dendrimer-based strategy provides a highly sensitive label free biosensor approach with over two times better detectability than conventional SPR. Both the biosensor device and the carrier structure hold great potential in clinical diagnosis for biomarker analysis in whole serum samples and other human biological samples.

Keywords: nanoplasmonic biosensor, gold nanodisks, dendrimer, allergy diagnosis, IgE quantification, serum clinical sample, label-free detection 


\section{Introduction}

Health and clinical care increasingly demand new modalities of diagnostic instrumentation which can satisfy strict requirements that conventional methods cannot provide. In this sense, decentralized reliable analysis with diagnostic platforms performed at patient bedside, or in primary care doctor's office, which are fast, sensitive and minimize sample manipulation is still needed. The development of these point-of-care (POC) devices could simplify disease diagnosis and could aid in following disease progression (or regression). Optical biosensors stand out among other configurations as the best candidates to fulfill POC requirements. They show exceptional potential for integration in portable lab-on-a-chip devices. They provide direct quantification of molecules in a real time and label-free manner (Estevez et al., 2012; Ligler, 2008). Plasmonic sensors and Surface Plasmon Resonance (SPR) sensor in particular are currently routine analytical instruments in laboratories due to its simplicity, robustness and versatility (Homola, 2008). Some of the well-known SPR limitations, such as moderate sensitivity, limited throughput capabilities and difficult miniaturization can be surpassed by using nanoplasmonic-based biosensors (Estevez et al., 2014; Petryayeva and Krull, 2011; Sepúlveda et al., 2009), whose configuration is based on the localized surface plasmon resonance (LSPR) rather than conventional SPR. Basically, the LSPR is the localized oscillation of free electron charges of metallic nanostructures with a resonance wavelength $\left(\lambda_{\text {LSPR }}\right)$ which is determined by their composition, size, shape, and the refractive index (RI) of the surrounding dielectrics. LSPR has emerged during last decade due to the eruption of a wide variety of plasmonic nanostructures. However despite its demonstrated potential, its applicability as biosensing devices has not been fully exploited and mainly proof-of-concepts are reported in the literature. Appropriate biofunctionalization of the nanostructures is crucial to take full advantage 
of such sensitive devices and the complexity of this step may lie behind the lack of reported complete bioanalysis in contrast with the high amount of examples based on conventional SPR. New biofunctionalization strategies which ensure stable and reliable assays, which in addition reduce potential nonspecific adsorption, are always sought. In particular, initial coatings or modification of the surface using functional linkers that in turns allows further specific receptor attachment have a direct influence on the final performance. Besides proteins, which are commonly used as carrier structures, dendrimers have profiled themselves as attractive materials because of the inherent capabilities conferred by their three-dimensional architecture (Astruc et al., 2010). Their structural homogeneity, controlled composition and multifunctionality make them unique for biomedical applications (Svenson and Tomalia, 2012), especially for drug delivery (Kesharwani et al., 2014) but also for biosensing (Fu and Li, 2013). Their use in immunochemistry has increased exponentially due to their interesting properties for the immobilization of biomolecules or small haptens. The globular-shaped poly(amido amine) PAMAM dendrimers (carrying amino groups in their structure) is the first and most extensively studied family (Trinchi and Muster, 2007). Conventional peptide carriers such as bovine serum albumin (BSA) or poly-L-lysine (PLL) usually lead to uncontrolled density and randomly distribution of the haptens in their structure, which can eventually induce reproducibility issues. In contrast, the regular geometry, stability, and high surface functionality of the dendrimers provide better control over the density and spacing of the immobilized molecules and also on the thickness of the overall receptor layer.

Herein, we propose an unprecedented approach that combines the advantages of a recently reported novel and highly sensitive label-free nanoplasmonic biosensor based on gold nanodisks with a generation $2\left(\mathrm{G}_{2}\right)$ BAPAD dendron-based structure in order to directly quantify specific 
antibodies in human samples. The optical label-free sensor is based on a novel waveguiding mechanism occurring in nanoplasmonic structures composed out of short-range ordered arrays of gold nanodisks (Otte et al., 2011) which results not only in higher sensitivities to RI changes, but also in improved signal to noise ratios, assuring an overall significant improvement of the RI sensor performance. We have designed a compact read-out setup for the real time evaluation of the biomolecular interactions occurring in the functionalized nanostructures.

Based on these grounds we have focused on the development of a highly sensitive and reproducible diagnostic tool for the quantitative detection of specific $\operatorname{IgE}$ antibodies in amoxicillin (AX) allergic patients. Current available diagnostic methods employed to assess subjects with an immediate allergic reaction include a complete clinical history plus in vivo skin tests or/and in vitro determination of specific IgEs in blood (Torres et al., 2003), whose production is triggered by the organism during an allergic outburst. Skin testing has been the most generalized approach. However this technique is invasive, painful (especially for children) and is not always appropriate particularly when the patient has suffered a severe allergic reaction. It requires the patient to temporarily stop medication, and results can be affected by skin condition. Solid phase immunoassays, such as the radioallergosorbent test (RAST) and subsequently the ImmunoCAP test have profiled themselves as best alternatives and are nowadays complementary in clinical diagnostics to the skin prick tests. Typically less sensitive than in-vivo skin tests (Chinoy et al., 2005), RAST is of a more qualitative nature and the results are usually expressed in a specific numerical scoring system (RAST scale from 0 to 6). On the other hand ImmunoCAP tests, based on fluorescent enzyme-labeled immunoassay (FEIA) (Mayorga et al., 2010), shows improved performance resulting from the high-binding capacity of the solid phase used to keep the allergen bound, which results in enhanced sensitivity. This 
technology has been cleared by the Food and Drug Administration (FDA) to provide quantitative measurements of $\operatorname{IgE}$ concentration in blood, and is a validated method with a working range of $0.35-100 \mathrm{kU} / \mathrm{L}(0.84-242 \mathrm{ng} / \mathrm{mL}$ ), and a cutoff value of $0.35 \mathrm{kU} / \mathrm{L}$ (Alonso et al., 1995; Blanca et al., 2001). In fact, RAST scores have been correlated with IgE concentration determined with ImmunoCAP tests. As alternative to these conventional techniques, PAMAM dendrimers have been used as carrier structure to conjugate $\beta$-lactam antibiotics mimicking the behavior of protein-hapten conjugates. They have been able to recognize specific IgEs (Sánchez-Sancho et al., 2002), attached to cellulose disks (Montañez et al., 2008) or to silica nanoparticles (Vida et al., 2013) and used as solid phase in RAST assays of serum patient samples. However none of these studies provided quantitative information of $\operatorname{IgE}$ concentration.

In this study we have developed not the whole dendrimer but a novel BAPAD-dendron structure as carrier molecule which can be easily transferred to (nano)plasmonic biosensor devices, allowing orthogonal coupling both to the gold surface and to amoxicillin molecules through the reactive amine groups in the outer shell. Using this strategy, very low concentrations of specific antibodies have been detected $(\mathrm{LoD}=0.6 \mathrm{ng} / \mathrm{mL})$, that is better than the one achieved with the ImmunoCAP test. The appropriate surface coating minimizes nonspecific interactions which enables direct whole serum evaluation. A validation of the assay has been done with real serum samples from hospital patients and the excellent correlation with ImmunoCAP allergy test confirms the reliability and viability of our approach as a potential tool for fast and real-time diagnosis of drug allergy. 


\section{Materials and Methods}

\subsection{Nanodisks preparation and optical setup description}

Short-ordered arrays of gold nanodisks $(\mathrm{D}=100 \mathrm{~nm}, \mathrm{H}=20 \mathrm{~nm}(\mathrm{Ti} / \mathrm{Au}=1 / 19 \mathrm{~nm}))$ were fabricated on glass substrates via hole-mask colloidal lithography (HCL) (Fredriksson et al., 2007). Before use, the substrates were cleaned by consecutive 1 min sonication cycles in acetone, ethanol and MilliQ water, dried with $\mathrm{N}_{2}$ stream and placed in an $\mathrm{UV} / \mathrm{O}_{3}$ cleaner tor for 20 min, after which they were rinsed with ethanol and water and dried with $\mathrm{N}_{2}$. Then, the substrates were clamped between a trapezoidal glass prism $(n=1.52)$ contacting the sample through RI matching oil $(\mathrm{n} \approx 1.512)$ and a custom-made Delrin flow cell (volume $=4 \mu \mathrm{L})$, which is connected to a microfluidic system consisting on a syringe pump (New Era, NE-1000) with adjustable pumping speed that ensured a constant liquid flow and a manually operated injection valve (IDEX Health and Science, V-451). The biosensing surfaces were excited by a collimated halogen light source (HL-2000, Micro-pack) set in TE polarization for gold nanodisks (LSPR mode). The light reaches the substrates through the prism and the reflected light is collected and fiber-coupled to a CCD spectrometer (Ocean Optics, Jazz Module). The setup design involves a triangular platform with lateral sides designed at an angle of $80^{\circ}$ with attached rails that incorporates the optical components, ensuring incoming and outgoing optical paths to remain constant at the desired angle (see Figure 1). Reflectivity spectra were acquired every 3 ms, and 300 consecutive spectra were averaged to provide the spectrum to be analyzed. Tracking of the real-time resonance peak position was achieved via polynomial fit using a homemade readout software. 


\subsection{Surface functionalization and IgE detection}

Reduction of the disulfide bond in the PMA-AX dimer $\left(0.25 \mathrm{mM}\right.$ in $\left.\mathrm{H}_{2} \mathrm{O}\right)$ was carried out by reaction with $10 \mathrm{mM}$ dithiothreitol (DTT; Sigma Aldrich) during 15 min under continuous agitation. The sample was immediately injected and flowed through the sensor surface at 15 $\mu \mathrm{L} /$ min keeping $\mathrm{H}_{2} \mathrm{O}$ as running buffer. Blocking of PLL-g-PEG was carried out by injecting 0.5 $\mathrm{mg} / \mathrm{mL}$ PLL(20)-g[3.5]-PEG(2) (SuSoS AG) in HEPES $10 \mathrm{mM}$. This immobilization step and all subsequent measurements were performed in situ, by continuously monitoring the signal response in real time.

After immobilization, PBST 0.5\% (PBS $+0.5 \%$ Tween 20) was settled as running buffer. For optimization and assessment studies, different concentration of monoclonal anti-penicillin IgG antibody (Acris Antibodies) were diluted in PBST 0.5\% and flowed over the functionalized surface at $25 \mu \mathrm{L} / \mathrm{min}$. Regeneration of the surface was achieved by injecting $20 \mathrm{mM} \mathrm{NaOH}$ at 65 $\mu \mathrm{L} / \mathrm{min}$. Total sample analysis were $30 \mathrm{~min}$, considering the surface regeneration step. Calibration curves were fitted to a saturation total binding model. LoD were calculated as the concentration corresponding to the blank signal plus three times its standard deviation (SD), while the LoQ were determined as the concentration corresponding to the minimum measurable signal, set as the blank signal plus 10 times SD.

\subsection{Real samples analysis}

Antifouling cocktail (AFC) used for serum measurements consisted of $10 \mathrm{mM}$ phosphate, 137 $\mathrm{mM} \mathrm{NaCl}, 2.7 \mathrm{mM} \mathrm{KCl}, 500 \mathrm{mM} \mathrm{MgCl} 2,2 \%$ Tween 20 and $2 \%$ of commercial human serum (Sigma-Aldrich). AFC buffer was kept in flow for 20 min before starting the measurements. Calibration curve for anti-penicillin monoclonal $\operatorname{IgG}$ in serum was performed using commercial human serum spiked with the antibodies. Calibration curve for anti-amoxicillin $\operatorname{IgE}$ was 
performed by serially diluting a serum sample with known concentration of specific IgE. Serum samples from allergic and non-allergic patients were extracted following standardized protocols by the Hospital de Málaga (Spain), and were analyzed with the ImmunoCAP test for amoxicillin IgE detection. Same clinical samples were directly flowed onto the functionalized surface with no previous pretreatment or dilution.

\section{Results and Discussion}

\subsection{BAPAD-AXO conjugate}

BisAminoalkyl PolyAmide Dendrimers (BAPAD) dendrimers and dendrons have been designed in order to emulate protein-like structures. The complete synthetic pathway is described in the Supporting Information and it can be summarized in Scheme 1. It is based on iterative reactions that allow the growth of the dendrimer structure, in this case, up to generation 2 . The strategy includes two steps: i) condensation between the core (cystamine), which incorporates the disulfide bridge that will be used to anchor to the gold surface, and the 3,3'-diazidopivalic acid (1) to obtain amide linked multi-azides; ii) reduction of the multi-azide structure to obtain the multi-amines, which then will be the reactive groups for the next generation of dendrimer. The strategy was repeated two times, in such a way that generation 2 (G2) dendrimers were obtained, having 8 amine groups in the outer shell. Then, with the aim of obtaining the dendrimericantigen functionalized with the allergenic determinant responsible of the allergic reaction to amoxicillin, this epitope was covalently bound to the dendrimer's periphery. D-BAPADG2 was reacted with amoxicillin at high $\mathrm{pH}$, to ensure that the amine groups at its periphery were unprotonated, acting as nucleophilic positions that efficiently attack the electrophilic carbonyl 
group of the beta-lactam antibiotic. Reaction conditions were optimized to obtain D-BAPADG2AXO with amoxicillin in all the reactive positions ( 8 antigenic determinants per structure).

Scheme 1. DBAPADG2 synthetic pathway and coupling of amoxicillin
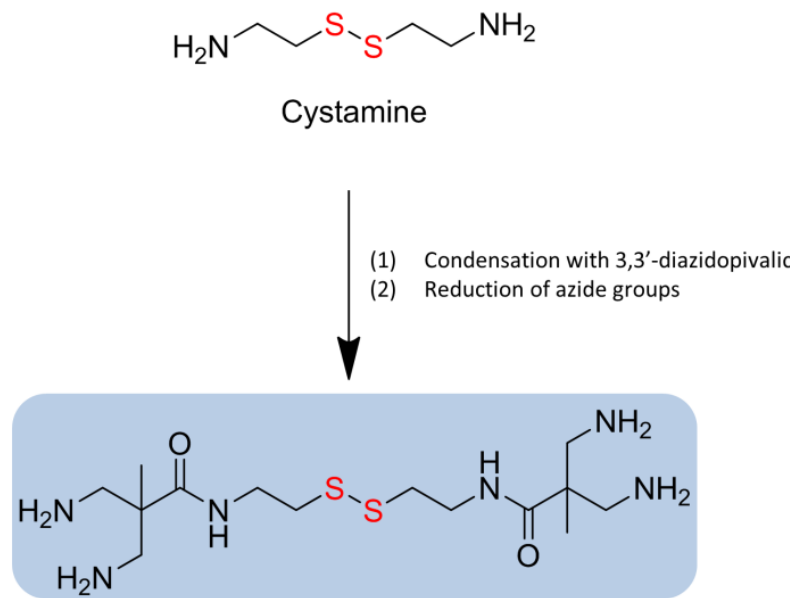

D-BAPADG1

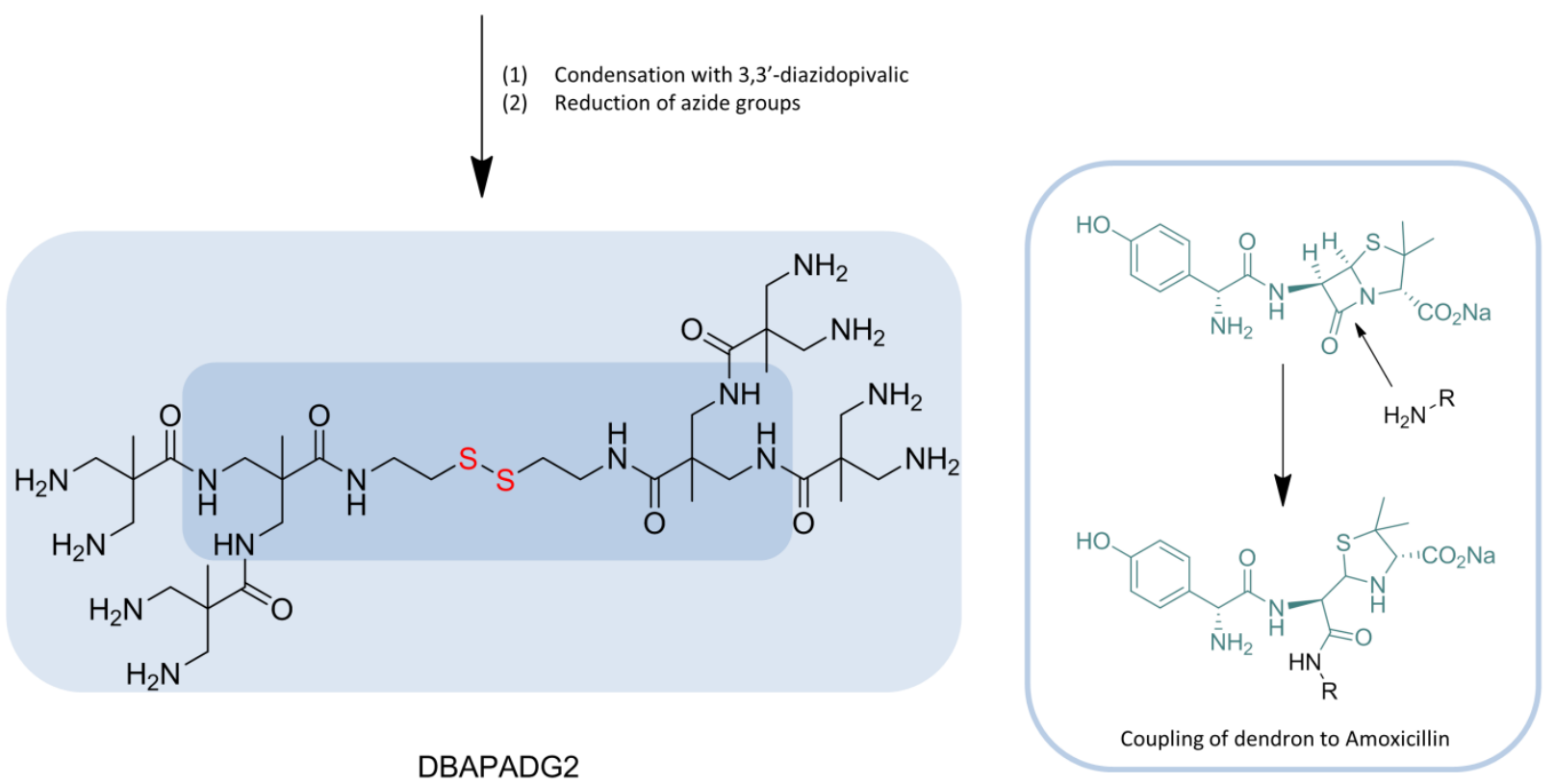




\subsection{Nanoplasmonic biosensor platform}

Short-range ordered arrays of gold nanodisks (diameter $\mathrm{D}=100 \mathrm{~nm}$, height $\mathrm{H}=20 \mathrm{~nm}$, surface density $\mathrm{F}=6-7 \%$ ) were fabricated by hole-mask colloidal lithography (HCL) (Fredriksson et al., 2007). In a recent study, we reported that these nanostructured substrates can sustain a novel inplane guided mode that can be excited in total internal reflection (Otte et al., 2011). Briefly, the in-plane LSPR excitation strongly enhances the polarizability of the nanodisks, creating an effective RI that is sufficiently large to support a guided electromagnetic mode inside this thin and sparse monolayer of nanostructures. This waveguided mode results not only in a large increase of the RI sensitivity, but also strongly improves the signal-to-noise ratio. Both effects assure an overall improved RI sensing performance that is up to an order of magnitude better than that of isolated non-interacting nanodisks. RI changes close to the nanostructure surfaces, such as the ones originating from biomolecular interactions are therefore much easier to detect. Hence, time-dependent monitoring of spectral LSPR peak displacements $\left(\Delta \lambda_{\text {LSPR }}\right)$ enables the extraction of real-time quantitative information related to the biomolecular interactions taking place (see Figure 1).

Previous in-house studies demonstrated that the resulting sensing performance of these guided modes depends on both the nanodisks surface density $(F)$ and the incidence angle of the light $(\theta)$ (Otte et al., 2011). Whereas the RI sensitivity increases as $\theta$ approaches 90 degrees, optimal mode excitation, which is characterized by light coupling efficiencies close to $100 \%$, strongly depends on the value of F. Hence, for an optimized sensing performance, F needs to be precisely chosen such that optimal mode excitation occurs at angles where the sensitivity is maximized, that is, angles close to $90^{\circ}$. However, due to imposed restrictions of our measurement setup (divergence of a finite-sized collimated broadband light source used for mode excitation), the 
largest possible incidence angle was restricted to $\theta=80^{\circ}$ degrees. For this particular angle, optimal mode excitation is achieved when the nanodisk surface density is approximately $\mathrm{F}=6$ 7\%. With this fact in mind, nanostructured substrates were fabricated with these surface occupancies, resulting in deep mode-specific reflectivity dips at $\lambda_{\mathrm{LSPR}} \approx 750 \mathrm{~nm}$. For these specific measurement conditions, a miniaturized system was devised, based on a triangular aluminum platform with lateral sides designed at an angle of $\theta=80^{\circ}$ (see Figure 1). This platform contains a custom-made flow cell and a specifically designed trapezoidal prism $\left(\theta=80^{\circ}\right)$. Additionally, optical rails that are perpendicularly attached to it, are used for the assembly of all optical components necessary for the TE mode excitation and the collection of the reflected light. The designed optical platform has very small dimensions (all the optical components are mounted on a $20 \times 20 \mathrm{~cm}$ portable breadboard), exemplifying its facile miniaturization and potential portability. Bulk sensitivity experiments confirmed the expected improved sensing performance at this high angle when compared to a similar setup mounted on a platform designed at an incidence angle of $\theta=70^{\circ}$. Specifically, an approximately 4 times better sensitivity $\left(\eta_{\mathrm{B} 80^{\circ}} \approx 375 \mathrm{~nm} \mathrm{RIU}{ }^{-1}\right.$ vs $\eta_{\mathrm{B} 70^{\circ}} \approx 100 \mathrm{~nm} \mathrm{RIU}{ }^{-1}$ ) was obtained (see Figure S1 in the Supporting Information, SI), confirming the enhanced sensitivity of the high-angle LSPR sensor platform. 

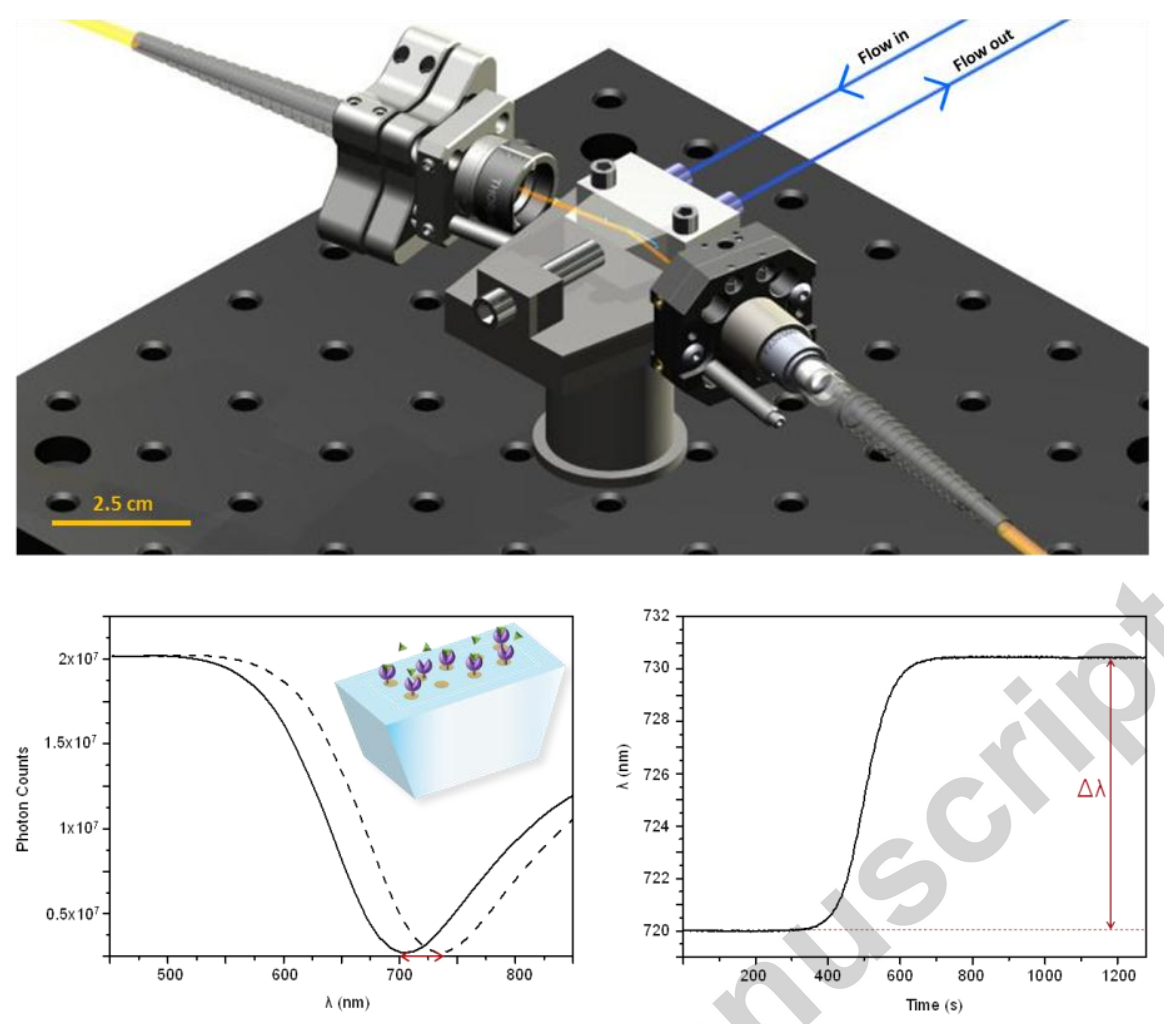

Figure 1. Top: Schematic of the miniaturized nanoplasmonic biosensor. Bottom: (left) graph showing the resonance peak (photon counts vs. $\lambda$ ); (right) graph showing the shift of the resonance peak over time ( $\Delta \lambda_{\text {LSPR }}$ of the minimum $v s$. time).

\subsection{Biosensor strategy: optimization and assessment}

In biosensing experiments, besides the inherent sensitivity imposed by the sensor, there are additional factors that are equally important to define the analytical sensitivity, the selectivity and the biosurface stability for a particular sensing assay. In our case, the achievement of an efficient immobilization onto the gold nanodisks is the most critical one. In general, surface modification of gold patterned surfaces requires conditions that assure specific and oriented binding onto the sensing areas (gold nanodisks) while avoiding non-specific binding of molecules to the substrate (glass). This can be achieved via a stepwise selective surface modification protocol with appropriate reagents, thereby exploiting the strong selectivity of gold for thiol-based compounds. For this reason, a dendron structure was designed and synthesized, 
with a spacer arm bearing a thiol group on the core and amoxicilloyl groups (AXO) in its outer shell (d-BAPADG2-AXO conjugate, see Scheme 1 and Figure 2). This design allows a fast and simple functionalization onto gold surfaces in only two steps: (i) the reduction of the disulfidecore of the dendrimer to create two thiol-functional symmetric dendrons, and (ii) their direct immobilization on the gold nanodisks by thiol chemisorption (see Figure 2). Using the dBAPADG2-AXO dendron, which incorporates a short thiol linker, a self-assembled layer is formed in a single-step chemisorption procedure, providing a well-ordered receptor layer over the gold nanodisks surface. In a separate step, $\mathrm{SiO}_{2}$ was independently modified by using poly-Llysine grafted PEG (PLL-g-PEG). This copolymer creates a brushed coating on $\mathrm{SiO}_{2}$ surfaces which has proven to successfully prevent and reduce nonspecific adsorption of proteins due to its hydrophilic properties (Marie et al., 2007; Soler et al., 2014). 
(a)

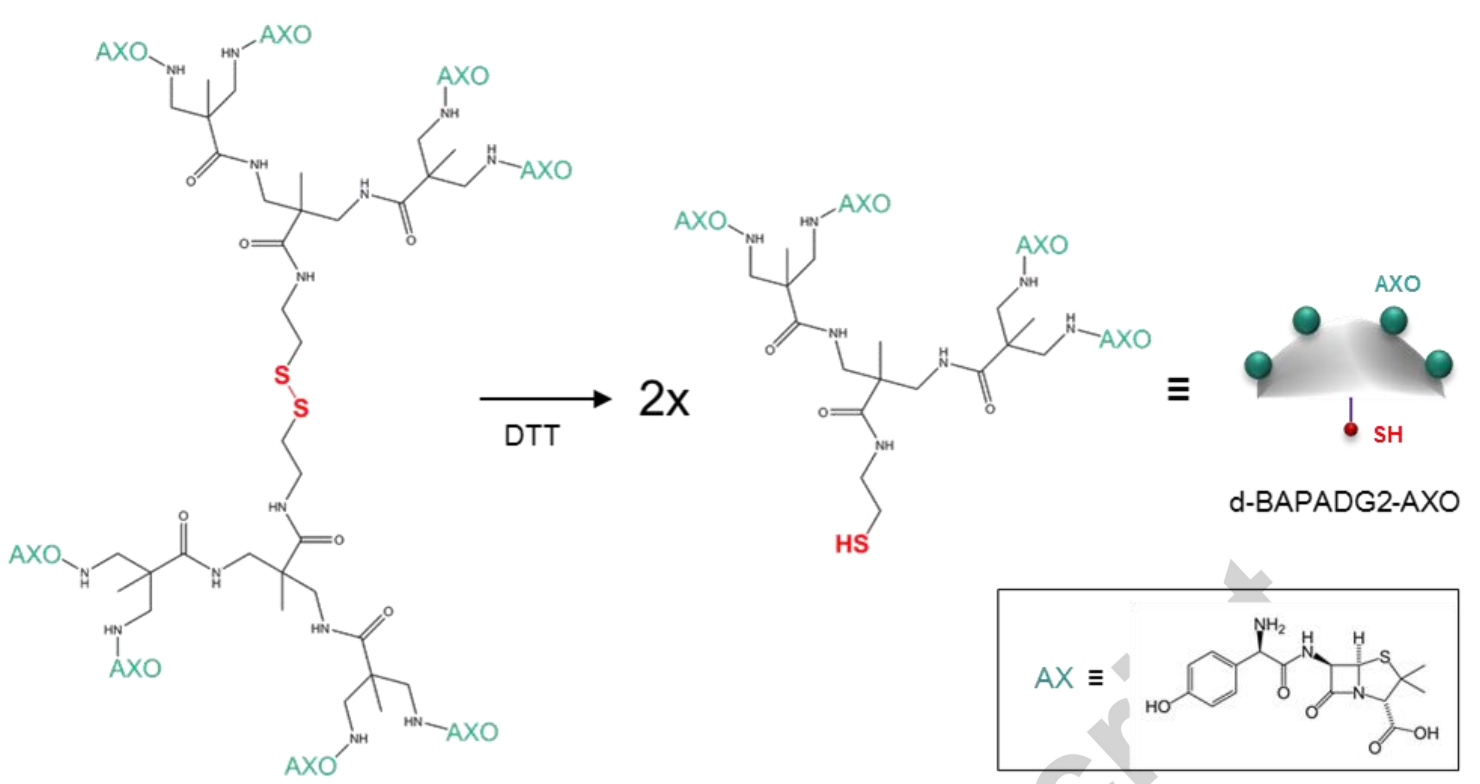

(b)

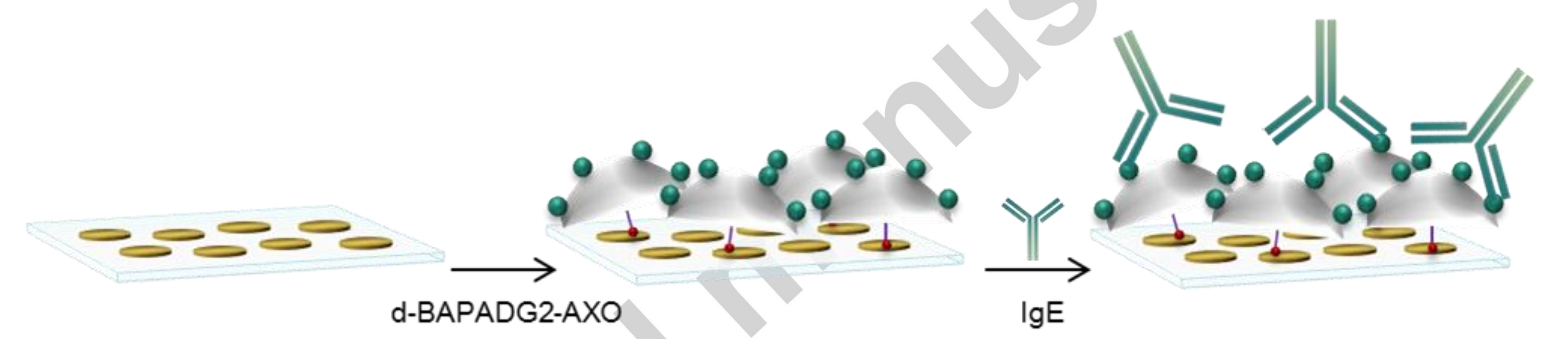

Figure 2. Schematic representation of the d-BAPADG2-AXO based biosensing strategy: (a) disulfide bond reduction with DTT and (b) direct immobilization on gold nanodisks by chemisorption and subsequent detection of specific IgE antibodies.

The structure of the amoxicillin-conjugated dendrimer d-BAPADG2-AXO was designed for the detection of $\operatorname{IgE}$ antibodies specific for this antibiotic. The $\mathrm{AX}$ was coupled via the $\beta$-lactam ring opening, in such a way that the rest of the structure particular to this penicillin (the thiazolidine ring and the variable side chain characteristic for AX) would remain exposed. Ideally in order to develop the assay (i.e. to generate and optimize a calibration curve) the appropriate target should be IgEs obtained from patients, or alternatively, $\operatorname{IgE}$ obtained after an external intended production process. However, the standard antibody production protocols are usually directed towards the mechanisms based on $\operatorname{IgG}$ generation, which are the most abundant types in serum. 
Given the difficulty of having specific IgE antibodies for amoxicillin, the evaluation and optimization of our biosensing approach was carried out with commercial anti-penicillin IgG antibodies. Although not exclusively specific for amoxicillin, but also for other $\beta$-lactam penicillins, these generic IgG antibodies are very useful to evaluate the behavior of the dBAPADG2-AXO coated surface and the viability of our proposed approach for detecting IgEs in serum.

The dendron structure incorporating the target molecule on its outer shell was directly attached to the gold surface in a single step. This step was performed in situ by injecting the solution of dBAPADG2-AXO through the cell. The immobilization was therefore continuously monitored in real time. Efficient and stable attachment was achieved, observing higher signals as the conjugate concentration increased (see Figure S2 in SI). The immobilization step is concentration dependent and shows high reproducibility (CV $\sim 5-6 \%$, see Table S1 in SI) reaching maximum surface coverage without affecting antigen accessibility at a concentration of $0.25 \mathrm{mM}$ (see Figure 3a). Higher concentrations did not translate into higher signal, which may be a sign of steric hindrance resulting from a high density of conjugates on the surface. Specificity studies done with other IgGs and control proteins (such as BSA) resulted in negligible binding onto the d-BAPADG2-AXO functionalized surface (see Figure 3b), indicating a high degree of selectivity of the active surface. Moreover, d-BAPAD-based strategy provides robust and long-time stable receptor surface because of the strong binding mechanism implicated in the gold functionalization (i.e. thiol chemisorption). Regeneration of the functionalized surface with basic conditions $(\mathrm{NaOH} 20 \mathrm{mM}$ ) led to complete removal of the recognized antibodies after each assay while retaining the integrity of the receptor layer with good levels of reproducibility for more than 40 cycles (an example of antibody detection and complete regeneration is shown 
in Figure $\mathrm{S} 3$ in the SI). Using the optimal d-BAPADG2-AXO concentration and IgG specific for amoxicillin a promising Limit of Detection (LoD) of $4 \mathrm{ng} / \mathrm{mL}\left(\mathrm{R}^{2}=0.9920\right)$ was determined. This detectability is better than the one obtained with a $70^{\circ}$ angle LSPR sensor $(7 \mathrm{ng} / \mathrm{mL})$ and the one reached with conventional SPR $(10 \mathrm{ng} / \mathrm{mL})$ using the same immunoreagents (see Figure S4 in the SI) which successfully confirms the better performance of our LSPR biosensor.

(a)

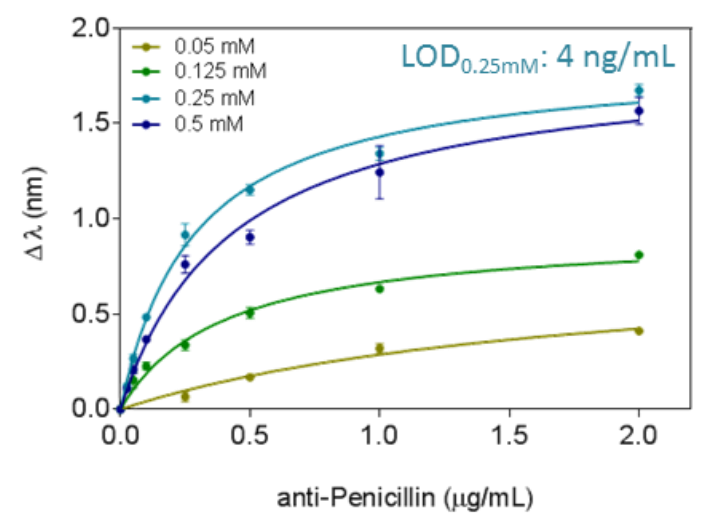

(b)

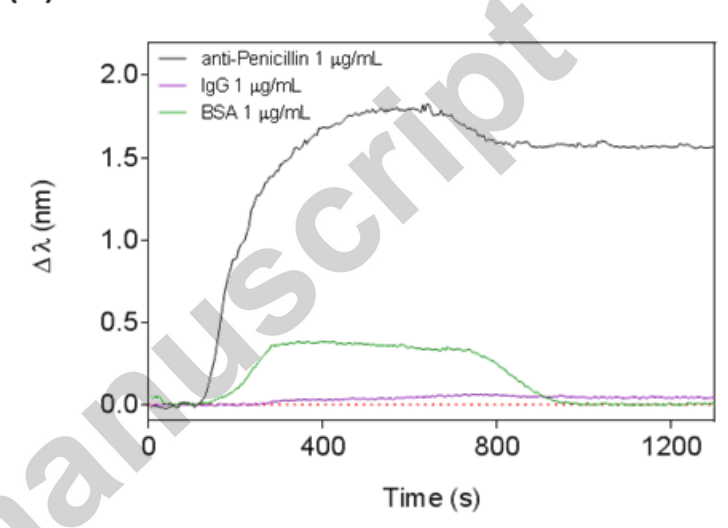

Figure 3. (a) Calibration curves for anti-penicillin detection at different d-BAPADG2-AXO concentrations $(0.05-0.5 \mathrm{mM})$ performed with $80^{\circ}$-nanoplasmonic sensor; (b) Specificity study for the d-BAPADG2-AXO based strategy: sensorgrams for the detection of specific antipenicillin antibody (black), nonspecific IgG antibody (purple) and bovine serum albumin (green) at $1 \mu \mathrm{g} / \mathrm{mL}$; red dashed line indicates reference (background signal). Assays performed with $80^{\circ}$ nanoplasmonic setup.

Overall, the design of the dendron structure represents a substantial step forward compared with the strategies used so far based on conventional dendrimers (Altintas et al., 2012; Mark et al., 2004; Pillet et al., 2013; Singh et al., 2009). In those cases, whole dendrimers were used as carriers in optical label-free biosensors, with the idea of increasing the immobilization capacity and enhancing the efficiency and stability of the modified surface. However, that approach requires extra steps to couple the dendrimers to the transducer surface (i.e. surface modification with a SAM and subsequent covalent attachment of the dendrimers) which considerably stretches 
on the whole process and can in turn affect the overall reproducibility of the assay. We performed a comparison using a G2-PAMAM peripherally decorated with AXO and covalently attached over a modified gold nanodisk surface (experimental data is summarized in the SI) and we observed: (i) lower immobilization efficiency with worse reproducibility (ii) worse overall sensitivity (almost $\sim 3$ times higher LoD) (Figure S5 and Table S1 in SI). In the case of dBAPADG2-AXO dendron, the thiol-directed attachment and oriented immobilization allows site-specific attachment in a more reproducible way than with the covalent binding, leaving exposed the AXO groups and maximizing antibody binding. This confirms the capabilities of the thiol-based d-BAPADG2-AXO dendron as a carrier scaffold.

\subsection{Human samples analysis}

The main goal of any biosensor is the demonstration of its applicability for the detection of a substance of interest in real samples, and if the intended use is in the clinical field, the purpose would be the direct analysis of human fluids. In the case of label-free optical biosensors where the signal comes indirectly from the mass adsorbed on the surface, the main challenge is to avoid nonspecific adsorption that can lead to false positive signals. There is a wide interest in developing biofunctionalization and assay strategies to prevent or minimize nonspecific binding onto the sensor surface. The use of PEGylated compounds together with the addition of surfactants to the buffer have proven to reduce the adsorption of proteins on different substrates (Krishnan et al., 2008). In this work, our goal was the analysis of serum samples from patients with allergy. We combined the use of PLL-g-PEG, that selectively binds to the glass and creates a hydrophilic layer which passivates the non-sensitive areas of the nanostructured substrates (Marie et al., 2007), with the use of an antifouling running solution that minimizes the nonspecific adsorption. Most common additives in antifouling cocktails are non-ionic detergents 
(e.g. Tween 20), due to their ability to disrupt ionic and hydrophobic biomolecule-surface bonds, or blocking agents such as BSA, non-fat dry milk, fish gelatin or whole serum. We performed a study of different buffer formulations in order to minimize the background signal produced by undiluted human serum, testing several concentrations of Tween 20 and different additives (BSA and common control serum). Best results were obtained when combining PLL-g-PEG coated surfaces and an antifouling cocktail consisting of PBS with $2 \%$ of Tween 20 and $2 \%$ of commercial serum as running fluid (see Figure 4). The background signal observed under these conditions was low enough and highly reproducible (standard deviation below $0.01 \mathrm{~nm}$ ) to permit reliable detection of antibodies in whole serum, therefore assuming a constant background for all measurements. The calibration curve was obtained by injecting undiluted commercial serum spiked with anti-penicillin IgG antibodies at different concentration $(0.025-$ $2 \mu \mathrm{g} / \mathrm{mL}$ ) (see Figure 5a). A LoD of $8 \mathrm{ng} / \mathrm{mL}$ was achieved, while the limit of quantitation (LoQ) was set at $16 \mathrm{ng} / \mathrm{mL}$. The LoD was slightly higher than for standard buffer conditions due to the contribution of the constant background. However the profile of the curve was analogous proving that serum matrix does not significantly affect the analysis performance as long as the background nonspecific signal is kept constant. 


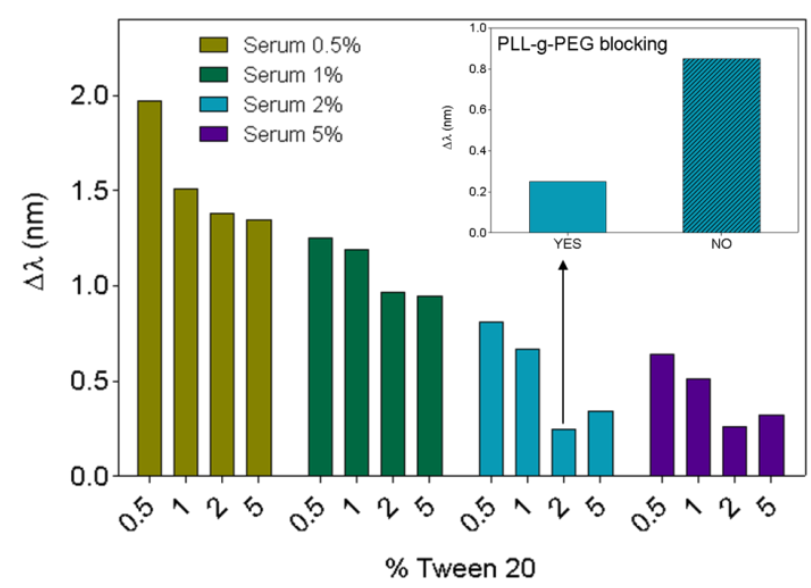

Figure 4. Background signal of whole serum onto the sensor surface blocked with PLL-g-PEG at different composition of running buffer: PBS + Tween $20(0.5-2 \%)+$ serum $(0.5-2 \%)$. Inset graph shows the influence of the PLL-g-PEG on the background signal by representing the nonspecific adsorption for the best running buffer conditions (PBS (2\% Tween 20 and $2 \%$ serum) on surfaces lacking PLL-g-PEG.

With these optimal conditions selected for the evaluation of whole serum, direct detection of real samples from patients was attempted. Due to the inherent differences between the IgGs used in the optimization step and the real target to measure (i.e. human specific IgE produced by the organism towards amoxicillin) we decided to perform a calibration curve directly with a serum sample from a patient, whose specific $\operatorname{IgE}$ concentration was known $([\operatorname{IgE}]=47.19 \mathrm{ng} / \mathrm{mL}$ determined with the ImmunoCAP assay). The sample was serially diluted with commercial serum and the curve was obtained using the previously optimized conditions for nonspecific adsorption minimization (see Figure 5b). Using IgEs as standard, a LoD of $0.6 \mathrm{ng} / \mathrm{mL}$ was achieved (more than one order of magnitude lower) and the LoQ resulted in $2.2 \mathrm{ng} / \mathrm{mL}$. This enhancement of the analytical sensitivity as compared to the previous values obtained with the anti-penicillin IgG (see Figure 5a) can be due to: (i) structural differences between IgG and IgE. IgE antibodies have a molecular weight that is approximately 1.3 times higher than IgGs (i.e. $\mathrm{MW}_{\mathrm{IgE}} \approx 200 \mathrm{kDa}, \mathrm{MW}_{\mathrm{IgG}} \approx 150 \mathrm{kDa}$ ), and since in plasmonic biosensors the mass plays a 
determinant role in the response, the output signal may be different; and (ii) a higher affinity for the antibodies produced by the human body against amoxicillin would be expected, as compared to the IgGs produced in mouse. Moreover, the IgG used as standard has a broad specificity towards the thiazolidine ring and, therefore, for several penicillins (i.e. it was produced using a conjugate of benzylpenicillin, presumably through $\beta$-lactam ring opening). Therefore, a lower affinity compared to the IgEs directly generated against amoxicillin would probably lead to lower signals at the same concentration. Overall, the improvement of the sensitivity when using the appropriate target clearly confirmed the higher natural affinity of the IgE against amoxicillin than the one of commercially produced IgG. 
(a)

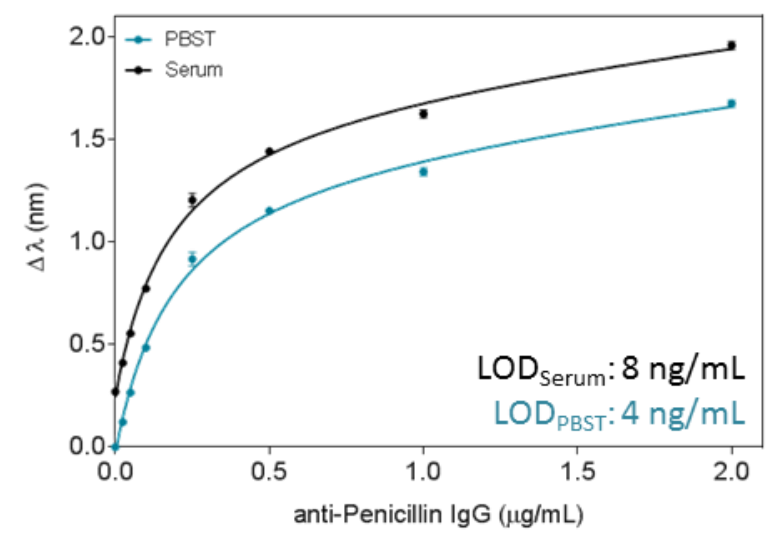

(b)

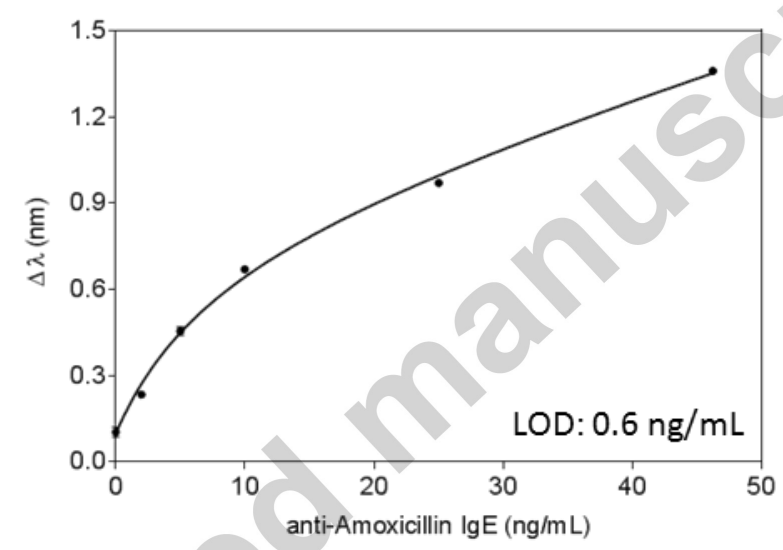

Figure 5. (a) Anti-penicillin IgG calibration curves in PBST (blue) and whole serum (black); (b) Amoxicillin-specific IgE calibration curve in serum samples.

The viability of the proposed biosensor methodology for allergy diagnosis was assessed by evaluating real clinical samples from allergic patients and quantified them using the $\operatorname{IgE}$ calibration curve. Non-pretreated and undiluted serum samples from amoxicillin allergic patients (S1, S3 and S5) and healthy donors (S2, S4 and S6) were directly flowed over the functionalized sensor surface by duplicate. Quantification of the specific IgE antibodies was carried out by interpolating the values of the signals in the calibration curve (see Figure 5b). Table 1 lists and 
compares the results of the analysis obtained with the nanoplasmonic biosensor (mean $\pm \mathrm{SD}$ ) and with the ImmunoCAP test performed at the hospital. All recoveries ranged between $96-115 \%$ evidencing good correlation between both diagnostic techniques. The concentration values obtained for each sample were also plotted and fitted to a linear regression function (see Figure 6). As can be observed, the excellent linearity $\left(\mathrm{R}^{2}=0.9988\right)$ and correlation between both approaches (slope $=1.173$ ) corroborate the accuracy of dendrimer-based nanoplasmonic biosensor to quantify $\operatorname{IgE}$ antibodies in whole serum.

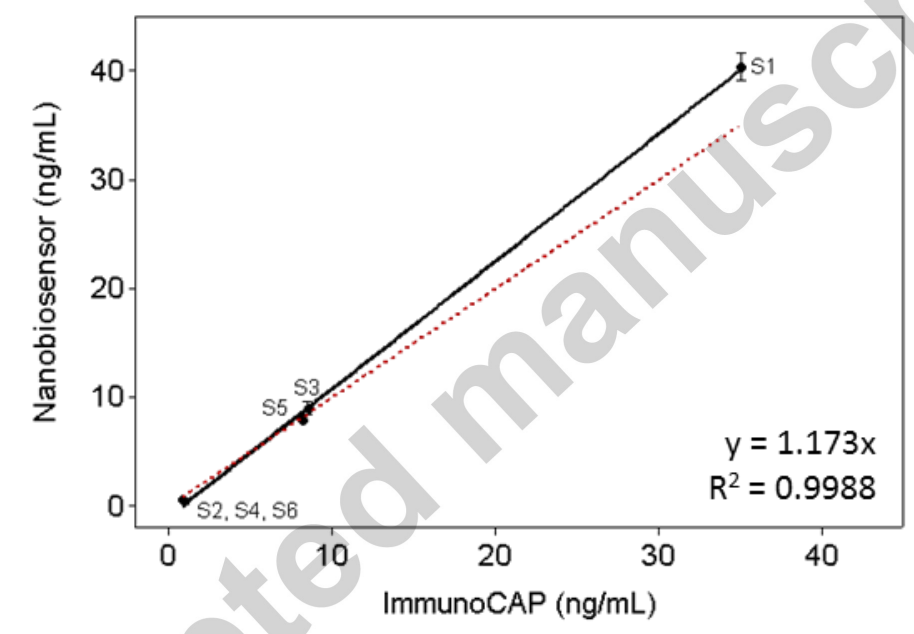

Figure 6. Accuracy studies performed with the nanoplasmonic biosensor. The graph shows the correlation between the values obtained with the sensing platform and the ImmunoCAP assay. Data shown correspond to the average of 2 replicates. Dotted line corresponds to a perfect correlation (slope $=1)$. 
Table 1. Clinical serum samples analysis determined by ImmunoCAP assay and the nanoplasmonic biosensor

\begin{tabular}{|c|c|c|c|c|c|}
\hline \multirow{2}{*}{\multicolumn{2}{|c|}{ Sample }} & \multicolumn{3}{|c|}{ anti-AX IgE Concentration } & \multirow{3}{*}{$\begin{array}{c}\text { \% Recovery } \\
115\end{array}$} \\
\hline & & \multirow{2}{*}{$\frac{\operatorname{ImmunoCAP}\left(\mathbf{k} \mathbf{U}_{\mathbf{I g E}} / \mathbf{L}\right)^{\mathbf{a}}}{14.5}$} & \multirow{2}{*}{$\frac{\text { ImmunoCAP }(\mathbf{n g} / \mathbf{m L})^{*}}{35.09}$} & \multirow{2}{*}{$\begin{array}{c}\text { Nanobiosensor }(\mathbf{n g} / \mathbf{m L}) \\
40.39 \pm 1.3\end{array}$} & \\
\hline S1 & $215 \mathrm{~F} 11$ & & & & \\
\hline S2 & $768 \mathrm{~F} 10$ & $\mathrm{ND}^{\mathrm{b}}$ & $\mathrm{ND}(<0.84)$ & $0.95 \pm 0.35$ & 113 \\
\hline S3 & $1103 \mathrm{~F} 09$ & 3.53 & 8.54 & $9.00 \pm 0.58$ & 105 \\
\hline S4 & $1732 \mathrm{~F} 09$ & ND & $\mathrm{ND}(<0.84)$ & $0.69 \pm 0.16$ & 100 \\
\hline S5 & $29 \mathrm{~F} 04$ & 3.4 & 8.23 & $7.87 \pm 0.27$ & 96 \\
\hline S6 & $2 \mathrm{~F} 06$ & ND & $\mathrm{ND}(<0.84)$ & $\mathrm{ND}(<0.6)$ & 100 \\
\hline
\end{tabular}

${ }^{a} 1 \mathrm{kU}$ IgE $/ \mathrm{L}=2.42 \mathrm{ng} / \mathrm{mL}$ (Winter et al., 2000)

${ }^{b} \mathrm{ND}$ : not detected (below detection limit of the assay of $0.35 \mathrm{kU} \mathrm{IgE}_{\mathrm{Ig}} / \mathrm{L}$ )

\section{Conclusions}

We present an innovative methodology for drug allergy diagnosis by direct quantification of amoxicillin specific IgE antibodies in serum using a novel nanoplasmonic biosensor device. We have designed and developed a complete biosensing strategy by combining specifically synthesized dendron-based amoxicillin conjugates with gold nanostructured surfaces leading to a fast, reliable, highly sensitive and label-free analysis. The thiol-functional dendron (dBAPADG2) allows simple and robust gold functionalization creating a regular and reproducible recognition layer. The d-BAPADG2-AXO dendron shows improved analytical features compared to PAMAM dendrimers in terms of sensitivity and surface reproducibility, achieving 2 times lower limits of detection. Moreover, the use of PLL-g-PEG for blocking the inert areas provides an antifouling coating that reduces the nonspecific adsorptions and minimizes the background signal when evaluating whole serum. The selectivity and reliability has been demonstrated by performing a calibration curve with undiluted serum samples spiked with antipenicillin IgG antibodies. Results showed that the serum components did not interfere in the antibody recognition process as similar sensitivity was achieved. To validate the proposed 
methodology a calibration curve was carried out with human specific amoxicillin IgE antibodies, revealing a notable increase of the sensitivity associated to the extremely high affinity of IgEs for the specific allergen. LoD was set at $0.6 \mathrm{ng} / \mathrm{mL}$ in whole serum. Finally, clinical samples from allergic patients and negative control samples were tested with the nanoplasmonic biosensor and the results were compared to those determined with an ImmunoCAP assay. An excellent accuracy was observed after obtaining recoveries in the range $96-115 \%$, with an exceptional linear correlation with the conventional immunoassay. The outstanding levels of sensitivity of the biosensor nanoplasmonic platform allow direct measurement of human samples without any kind of pretreatment or preconcentration of the sample with a total analysis time of $15 \mathrm{~min}$. The excellent features of the nanoplasmonic biosensor and the dendron-based conjugates represent an attractive alternative as a fast and user-friendly diagnostic tool for clinical analysis and for obtaining quantitative data of amoxicillin allergy. Moreover, the overall strategy can be virtually adapted to detect other clinical biomarkers or any kind of target, simply by conjugating them to the dendron structure. The presented biosensing methodology holds great promise to improve biomarkers detection directly in human samples and constitutes a potentially useful tool for clinical diagnosis practice and for the development of point-of-care devices.

\section{Acknowledgements}

M. Soler and P. Mesa-Antunez acknowledges financial support from "Programa de Formación de Personal Investigador (FPI)" from the Spanish Ministry of Economy and Competitiveness. The authors acknowledge the Red De Investigación de Reacciones Adversas a Alergenos y Fármacos (RIRAAF) (RD12/0013/0003), the Consejería de Salud from Junta de Andalucía (PI-0159-2013), the former Spanish Ministry of Science and Innovation (CTQ2010-20303) and the Spanish Ministry of Economy and Competitiveness (MULTIBIOPLAS project, TEC2099-08729) for the 
financial support. ICN2 is the recipient of Grant SEV-2013-0295 from the "Severo Ochoa Centers of Excellence" Program of Spanish MINECO.

Supporting Information. Detailed information about the synthesis of the D-BAPADG2-AXO and its characterization, and other experimental details related to biosensing strategies and the evaluation study are included in Supporting Information. This material is available free of charge via the Internet at http://www.sciencedirect.com

\section{References}

Alonso, R., Botey, J., Pena, J.M., Eseverri, J.L., Marín, A., Ras, R.M., 1995. J. Investig. Allergol. Clin. Immunol. 5(3), 156-160.

Altintas, Z., Uludag, Y., Gurbuz, Y., Tothill, I., 2012. Anal. Chim. Acta 712(0), 138-144.

Astruc, D., Boisselier, E., Ornelas, C., 2010. Chem. Rev. 110(4), 1857-1959.

Blanca, M., Mayorga, C., Torres, M.J., Reche, M., Moya, C., Rodriguez, J.L., Romano, A., Juarez, C., 2001. Allergy 56(9), 862-870.

Chinoy, B., Yee, E., Bahna, S., 2005. Clin. Mol. Allergy 3(1), 4.

Estevez, M.C., Alvarez, M., Lechuga, L.M., 2012. Laser Photon. Rev 6(4), 463-487.

Estevez, M.C., Otte, M.A., Sepulveda, B., Lechuga, L.M., 2014. Anal. Chim. Acta 806, 55-73. Fredriksson, H., Alaverdyan, Y., Dmitriev, A., Langhammer, C., Sutherland, D.S., Zäch, M., Kasemo, B., 2007. Adv. Mater. 19(23), 4297-4302.

Fu, C., Li, J.-P., 2013. Chin. J. Anal. Chem. 41(11), 1762-1772.

Homola, J., 2008. Chem. Rev. 108(2), 462-493.

Kesharwani, P., Jain, K., Jain, N.K., 2014. Prog. Polym. Sci. 39(2), 268-307.

Krishnan, S., Weinman, C.J., Ober, C.K., 2008. J. Mater. Chem. 18(29), 3405-3413.

Ligler, F.S., 2008. Anal. Chem. 81(2), 519-526.

Marie, R., Dahlin, A., Tegenfeldt, J., Höök, F., 2007. Biointerphases 2(1), 49-55.

Mark, S.S., Sandhyarani, N., Zhu, C., Campagnolo, C., Batt, C.A., 2004. Langmuir 20(16), 6808-6817.

Mayorga, C., Sanz, M., Gamboa, P., García, B., SEAIC, on behalf of the Clinical Immunology Committee of the Spanish Society of Allergology and Clinical Immunology of the SEAIC., 2010. J. Investig. Allergol. Clin. Immunol. 20(2), 103-109.

Montañez, M.I., Perez-Inestrosa, E., Suau, R., Mayorga, C., Torres, M.J., Blanca, M., 2008. Biomacromolecules 9(5), 1461-1466.

Otte, M.A., Estévez, M.C., Regatos, D., Lechuga, L.M., Sepúlveda, B., 2011. ACS Nano 5(11), 9179-9186.

Petryayeva, E., Krull, U.J., 2011. Anal. Chim. Acta 706(1), 8-24.

Pillet, F., Sanchez, A., Formosa, C., Séverac, M., Trévisiol, E., Bouet, J.-Y., Anton Leberre, V., 2013. Biosens. Bioelectron. 43(0), 148-154.

Sánchez-Sancho, F., Pérez-Inestrosa, E., Suau, R., Mayorga, C., Torres, M.J., Blanca, M., 2002. Bioconjug. Chem. 13(3), 647-653. 
Sepúlveda, B., Angelomé, P.C., Lechuga, L.M., Liz-Marzán, L.M., 2009. Nano Today 4(3), 244-251.

Singh, P., Onodera, T., Mizuta, Y., Matsumoto, K., Miura, N., Toko, K., 2009. Sens. Actuators B. Chem. 137(2), 403-409.

Soler, M., Estevez, M.-C., Alvarez, M., Otte, M., Sepulveda, B., Lechuga, L., 2014. Sensors 14(2), 2239-2258.

Svenson, S., Tomalia, D.A., 2012. Adv. Drug Deliv. Rev. 64, Supplement(0), 102-115.

Torres, M.J., Blanca, M., Fernandez, J., Romano, A., de Weck, A., Aberer, W., Brockow, K., Pichler, W.J., Demoly, P., for ENDA and the EAACI interest group on drug hypersensitivity., 2003. Allergy 58(10), 961-972.

Trinchi, A., Muster, T.H., 2007. Supramol. Chem. 19(7), 431-445.

Vida, Y., Montanez, M.I., Collado, D., Najera, F., Ariza, A., Blanca, M., Torres, M.J., Mayorga, C., Perez-Inestrosa, E., 2013. J. Mater. Chem. B 1(24), 3044-3050.

Winter, W.E., Hardt, N.S., Fuhrman, S., 2000. Arch. Pathol. Lab. Med. 124(9), 1382-1385.

\section{Highlights}

- A thiol-ending dendron has been specifically designed as carrier molecule

- Amoxicillin(AX)-coated dendron used as receptor in a novel nanoplasmonic biosensor

- Direct label-free quantification of AX specific IgEs in serum using the biosensor

- Antifouling surface conditions allow whole patient serum analysis without dilution

- Validation with standard immunoassay and real patient samples confirms the accuracy 